\title{
Caractéristiques de la flore et de la végétation ligneuses des parcs agroforestiers de l'arrondissement de Tendouck (Basse Casamance, Sénégal)
}

\author{
Ismaila COLY ${ }^{1 *}$, Tahirou Charles DIATTA ${ }^{1}$, Daouda NGOM $^{2}$, Aliou BADJI ${ }^{1}$ et \\ Omar GUEYE ${ }^{1}$
}

\author{
${ }^{1}$ Université Assane SECK, Laboratoire d'Agroforesterie et d'Écologie (LAFE), Département d'Agroforesterie, \\ UFR Sciences et Technologies, BP 523, Ziguinchor, Sénégal. \\ ${ }^{2}$ F S T/UCAD, Département de Biologie Végétale, Laboratoire d'Ecologie végétale, BP 5 005, Sénégal. \\ *Auteur correspondant ; E-mail : icoly@univ-zig.sn; Tel :00221775509534
}

\section{RESUME}

L'agriculture occupe une place très importante dans l'arrondissement de Tendouck. Pour augmenter leurs rendements, les agriculteurs laissent délibérément dans leurs champs certaines espèces ligneuses. Cette étude a pour objectif général de contribuer à une meilleure connaissance des caractéristiques des parcs agroforestiers du département de Bignona. Pour ce faire, une placette de $2500 \mathrm{~m}^{2}$ (50 mx50 m) a été installée dans les champs de chaque agriculteur retenu dans un échantillon de 98 agriculteurs. Cet échantillon est réparti entre les différentes communes de l'arrondissement au prorata de leur poids démographique soit $16 ; 21 ; 22 ; 30$ et 9 placettes respectivement dans les communes de Balinghore, Diégoune, Kartiack, Mangagoulack et Mlomp. La flore dans l'arrondissement de Tendouck est riche de 53 espèces réparties en 45 genres relevant de 20 familles botaniques. Au niveau de l'arrondissement de Tendouck la densité des arbres des parcs agroforestiers est de 42 pieds/ha, la surface terrière de $3,21 \mathrm{~m}^{2} /$ ha et le taux de recouvrement de $18,82 \%$. Ces parcs sont très diversifiés avec un indice de Shannon de 3,91 et un indice de Pielou de 0,79. Le taux de régénération y est très important $(88,19 \%)$. La structure par classes de hauteur du peuplement ligneux des parcs agroforestiers révèle une prédominance des jeunes individus avec 24,73\% des individus appartenant à la classe de hauteur [2-4 m]. La classe de diamètre la plus représentée est [5-20 cm] avec 42, 99\% des individus. Ces résultats constituent une base pour une gestion rationnelle et durable de ces écosystèmes en vue de permettre aux agriculteurs de mieux tirer profit de leurs services dans l'arrondissement de Tendouck. Il apparait ainsi judicieux de poursuivre cette étude dans les autres départements de la Basse Casamance en vue de disposer d'une base de données des parcs agroforestiers de cette zone éco géographique.

(C) 2020 International Formulae Group. All rights reserved.

Mots clés: Champs, composition floristique, structure, agriculteurs, caractéristiques structurales

\section{Characteristics of the woody flora and vegetation in the agroforestry parks of the Tendouck district (Lower Casamance, Senegal)}

\begin{abstract}
ABSRACT
Agriculture occupies a very important place in the district of Tendouck. In order to increase their yields, farmers deliberately leave certain woody species in their fields. The general objective of this study is to contribute
\end{abstract}


to a better knowledge of the characteristics of agroforestry parks in the Bignona district. To this end, a $2500 \mathrm{~m}^{2}$ $(50 \mathrm{mx} 50 \mathrm{~m})$ plot was installed in the fields of each farmer selected from a sample of 98 farmers. This sample is distributed among the different communes of the district in proportion to their demographic weight, therefore $16 ; 21 ; 22 ; 30$ and 9 plots respectively in the communes of Balinghore, Diégoune, Kartiack, Mangagoulack and Mlomp. The flora in the district of Tendouck is rich of 53 species divided into 45 genera belonging to 20 botanical families. At the level of Tendouck district, the density of trees in agroforestry parks is 41.55 trees/ha, the basal area of $3.21 \mathrm{~m}^{2} / \mathrm{ha}$ and the rate of covering of $18.82 \%$. These parks are very diversified with a Shannon index of 3.91 and a Pielou index of 0.79 . The regeneration rate is very high $(88.19 \%)$. The height class structure of the woody stand in the agroforestry parks reveals a predominance of young individuals with $24.73 \%$ of the individuals belonging to the height class [2-4 m]. The most represented diameter class is [5-20 cm] with $42.99 \%$ of the individuals. These results provide a basis for rational and sustainable management of these ecosystems with a view to improving the living conditions of farmers in the Tendouck district. It therefore seems advisable to continue this study in the other departments of Lower Casamance in order to have a data of the agroforestry parks of this eco-geographical area.

(C) 2020 International Formulae Group. All rights reserved.

Keywords: Fields, floristic composition, structure, farmers, structural features

\section{INTRODUCTION}

En Afrique de l'ouest l'agriculture de subsistance est marquée par une conservation du système traditionnel d'utilisation des terres, celui des «parcs agroforestiers» où des arbres parsèment les champs cultivés. Les agriculteurs conservent les arbres peu gênants ou utiles qui sont considérés comme partie intégrante du système de culture (Boffa, 2000; Smektala et al. 2005). Dans ce système, l'arbre joue un rôle important sur le plan agroécologique, socioéconomique et sanitaire. Ces parcs sont exploités par les populations qui y tirent des revenus additionnels à travers les nombreux produits fournis par les arbres tels que les produits alimentaires, médicinaux, bois de chauffe etc. (Badiane et al. 2019). Ils contribuent à $75 \%$ de la production de produits forestiers ligneux et non ligneux dans certaines régions d'Afrique de l'Ouest (Boffa, 2000).

Le Sénégal n'est pas épargné par cette situation et particulièrement la Basse Casamance. Cette zone où l'agriculture est essentiellement pluviale et saisonnière (Bassène, 2008) est caractérisée par une faible disponibilité des terres arables liée à une forte pression démographique et une dégradation des terres résultante de pratiques agricoles inappropriées. Ainsi, les agriculteurs font recours à la reconversion des forêts communautaires en champs de culture. Toutefois, ils conservent dans ces systèmes agraires les espèces ligneuses présentant un intérêt socio-économique ou agronomique formant ainsi des parcs agroforestiers qui constituent la plus grande partie du système agraire en Basse Casamance (Zoundi et Léonidas, 2003).

Malgré l'importance de ces parcs agroforestiers, ces formations végétales restent à nos jours très peu étudiées notamment dans le département de Bignona situé dans la partie Nord de la Basse Casamance. Ainsi pour mieux appréhender ces écosystèmes en vue de proposer des stratégies de leur gestion durable, nous nous sommes fixé comme objectif de caractériser la végétation ligneuse de ces parcs en évaluant leurs caractéristiques floristiques et structurales.

\section{MATERIEL ET METHODES \\ La zone d'étude}

L'étude a été menée en Basse Casamance plus particulièrement dans l'arrondissement de Tendouck qui appartient au département de Bignona et à la région de Ziguinchor. Cet arrondissement compte six communes dont cinq rurales (Kartiack, Mlomp, Diégoune, Mangagoulack et Balinghore) et une commune urbaine (Thionck Essyl) (Figure 1).

Selon la classification de Sagna (2005), la Basse Casamance appartient au domaine climatique sud-soudanien côtier. Elle 
correspond à la zone la plus pluvieuse du Sénégal (Sané, 2017) avec une pluviométrie moyenne annuelle de $1352,5 \mathrm{~mm}$ pour les trente dernières années. Le régime thermique de la région se caractérise par une température moyenne annuelle d'environ $27^{\circ} \mathrm{C}$ (ANACIM, 2019).

Le relief de la Basse Casamance présente deux grands ensembles (Goudiaby, 2013). Le premier est le plateau continental qui se caractérise d'une part par des sols ferrugineux tropicaux lessivés et d'autre part par des sols ferrallitiques faiblement à moyennement désaturés. Le second correspond au terres inondables avec deux faciès; les vallées rizicultivables juxtaposées aux forêts de mangrove.

Ce type de relief laisse apparaître une formation végétale constituée de savane, de forêts denses sèches de forêts galeries sur le plateau et de la mangrove représentée par des espèces comme Rhizophora racemosa G.Mey et Avicennia germinans L. sur les terres inondables (Mané, 2007).

\section{Relevés de la végétation ligneuse}

Un échantillonnage a été effectué pendant l'hivernage, au mois d'août 2018 au niveau des parcs agroforestiers de 10 villages répartis entre les (5) communes rurales de l'arrondissement, en considérant les cinq (5) communes rurales comme strates. Le choix des villages est basé sur le critère de la répartition géographique en vue d'assurer un bon maillage de chaque commune. Au niveau de ces villages, 98 placettes carrées de $2500 \mathrm{~m}^{2}(50 \mathrm{~m}$ de côté) ont été installées dans les champs des agriculteurs en raison d'une placette par agriculteur retenu. Le critère de choix des agriculteurs est la détention d'un champ cultivé. Une allocation proportionnelle des placettes aux différentes communes puis aux différents villages constitutifs de l'échantillon a été effectuée sur la base de leur poids démographique (Tableau 1). Ainsi, $16 ; 21$; 22 ; 30 et 9 placettes ont été installées respectivement dans les communes de Balinghore, Diégoune, Kartiack, Mangagoulack et Mlomp (Tableau 1).
Dans chaque placette, toutes les espèces ont été inventoriées et les paramètres dendrométriques des individus ayant un diamètre au tronc supérieur à $5 \mathrm{~cm}$ ont été mesurés. La hauteur des arbres a été mesurée à l'aide d'un dendromètre sunto ; le diamètre du tronc à hauteur de poitrine (DHP) à l'aide d'un compas forestier et le diamètre croisé du houppier (Est-Ouest et Nord-Sud) à l'aide d'un ruban métrique. La liste floristique a été établie sur la base de la flore du Sénégal (Berhaut, 1967). Les synonymes ont été actualisés sur la base de l'Enumération des plantes à fleurs d'Afrique Tropicale (Lebrun et Stork, 1997). La régénération de chaque espèce a été évaluée par un comptage exhaustif des individus de diamètre inférieur à $5 \mathrm{~cm}$.

\section{Le traitement des données}

Les données obtenues à partir des relevés de végétation ont été traitées à l'aide du tableur Excel qui a servi au classement des données numériques et à l'élaboration des tableaux et graphiques. Pour la discrimination des différents faciès rencontrés, les données d'inventaire dans les différentes communes ont été soumises à une analyse multivariée notamment l'Analyse en Composantes Principales (ACP) à l'aide du logiciel XLSTAT Version 2014.

Les formules ci-après ont été utilisées pour le calcul de certains paramètres de la végétation :

La richesse spécifique: la richesse spécifique totale (S) est le nombre total d'espèces que comporte le peuplement considéré dans un écosystème donné (Ramade, 2003 cité par Ngom et al., 2013).

L'analyse fréquentielle est une méthode qui consiste à apprécier la distribution des espèces à travers les relevés. La fréquence de présence renseigne sur la distribution d'une espèce dans un peuplement. Elle peut être exprimée en valeur absolue ou en pourcentage (\%). En \%, elle est estimée par la formule suivante (Roberts-Pichette et Gillespie, 1999). $F=\frac{N r i}{N r} * 100$

$\mathrm{F}=$ fréquence de présence exprimée en pourcentage $(\%) ; \mathrm{Nri}=$ nombre de relevés où 
l'on retrouve l'espèce i et $\mathrm{Nr}=$ nombre total de relevés.

La densité est le nombre d'individus par unité de surface. Elle s'exprime en nombre d'individus/ha. La densité observée ou densité réelle est obtenu par le rapport de l'effectif total des individus dans l'échantillon $(\mathrm{N})$ par la surface échantillonnée (S). $\quad D o b=N / S$

Le couvert ligneux est la surface de la couronne de l'arbre projetée verticalement au sol. Il est exprimé en mètre carré par hectare $\left(\mathrm{m}^{2} \cdot \mathrm{ha}^{-1}\right)$. Le couvert ligneux est calculé avec la formule suivant: $\quad C=\sum \pi\left[\frac{d m h}{2}\right]^{2} / S E$ Avec $\mathrm{C}=$ couvert ligneux $; \mathrm{dmh}=$ diamètre moyen du houppier en $\mathrm{m} ; \mathrm{SE}=$ surface de l'échantillon considéré en ha.

La surface terrière désigne la surface de l'arbre évaluée à la base du tronc de l'arbre. Elle est exprimée en mètre carré par hectare $\left(\mathrm{m}^{2}\right.$.ha. $\left.{ }^{-1}\right)$. Elle est donc obtenue à partir de la formule suivante : $S t=\sum \pi\left[\frac{d 1,3}{2}\right]^{2} / S E$
Avec $\mathrm{St}=$ surface terrière $; \mathrm{d} 0,3=$ diamètre en $\mathrm{m}$ du tronc à $1,3 \mathrm{~m}$; $\mathrm{SE}=$ surface de l'échantillon considéré en ha

L'indice de diversité (H') de Shannon Weaver (1949) est le plus couramment utilisé. Il exprime l'importance relative du nombre d'espèces abondantes dans un milieu donné. L'Indice de Shannon- Weaver, exprimé en bits (ou en Sha selon les auteurs), est donné par la formule suivante: $H^{\prime}=-\sum p i \log 2 p i$

$\mathrm{Pi}=$ abondance relative de chaque espèce. $\log$ (2) = logarithme calculé avec comme base 2. Pi $=\mathrm{Ni} / \mathrm{N}$ avec $\mathrm{Ni}=$ l'effectif de l'espèce $\mathrm{i} ; \mathrm{N}=$ effectif total des espèces

L'indice de régularité (E) renseigne sur la distribution des abondances des espèces dans le peuplement. Il est compris entre 0 et 1 . Il tend vers 0 quand l'ensemble des individus correspond à une seule espèce. Il tend vers 1 lorsque chacune des espèces est représentée par le même nombre d'individus (Ramade, 2009). Cet indice est donné par la formule suivante : $E=H^{\prime} / H^{\prime} \max$ avec Hmax $=\log 2(\mathrm{~S})$ avec $\mathrm{S}$ $=$ effectif total des espèces.

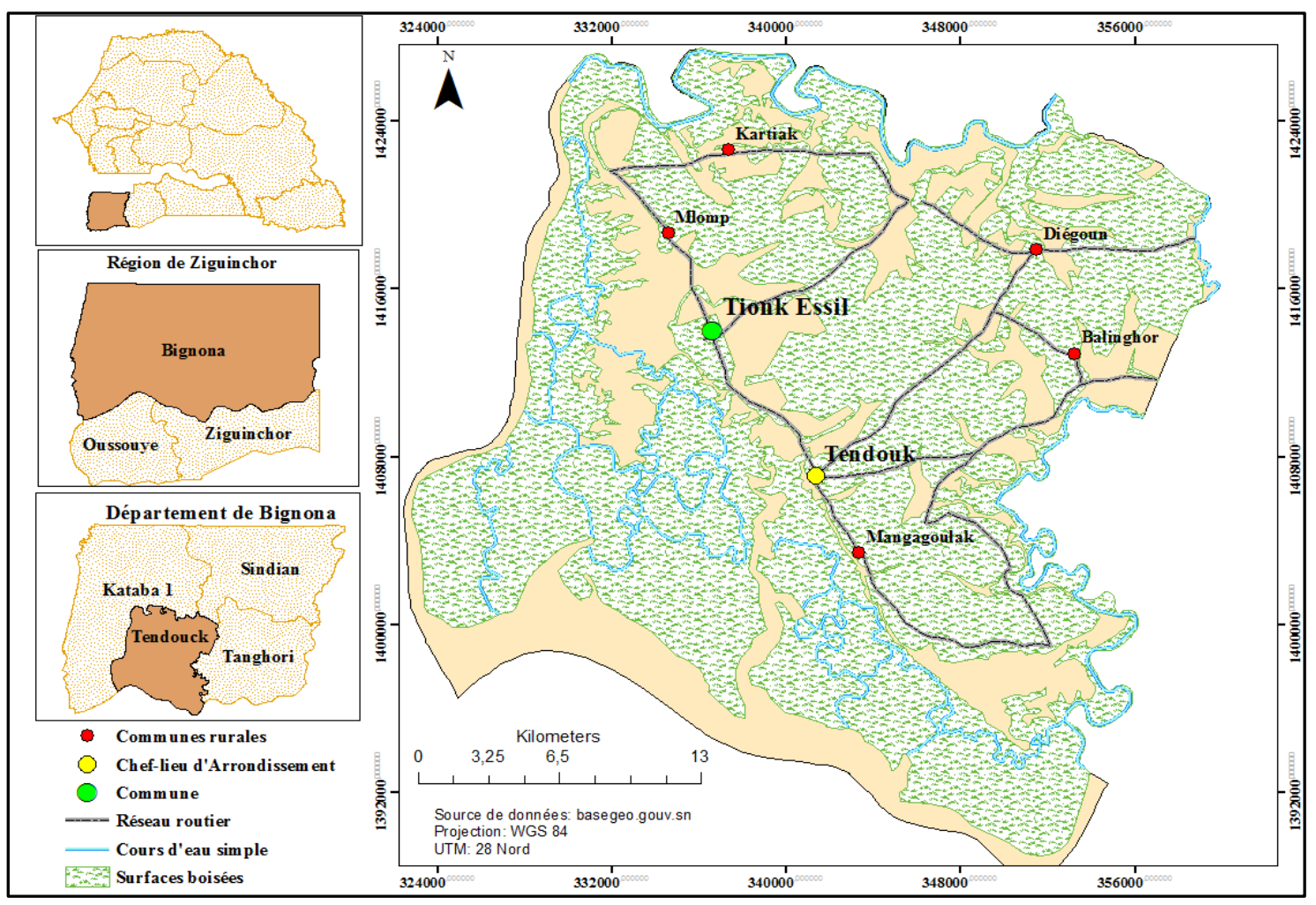

Figure 1: Carte de l'Arrondissement de Tendouck. 
Tableau 1 : répartition du nombre de villages et d'exploitations agricoles selon les différentes communes de l'arrondissement de Tendouck et dans l'échantillon.

\begin{tabular}{lcccc}
\hline & $\begin{array}{c}\text { Nombre de } \\
\text { villages }\end{array}$ & $\begin{array}{c}\text { Nombre d'exploitations } \\
\text { agricoles }\end{array}$ & $\begin{array}{c}\text { Nombre } \\
\text { de villages }\end{array}$ & $\begin{array}{c}\text { Nombre de } \\
\text { placettes }\end{array}$ \\
\hline Balinghore & 3 & 757 & 2 & 16 \\
Diégoune & 3 & 962 & 2 & 21 \\
Kartiack & 4 & 1014 & 2 & 22 \\
Mangagoulack & 7 & 1373 & 3 & 30 \\
Moulomp & 2 & 438 & 1 & 9 \\
\hline Total & 19 & 4544 & 10 & 98 \\
\hline
\end{tabular}

Chaque placette correspond à une exploitation agricole retenue dans l'échantillon.

\section{RESULTATS}

\section{Composition floristique}

La flore ligneuse des parcs de l'arrondissement de Tendouck est riche de 53 espèces. Celles-ci sont réparties en 45 genres appartenant à 20 familles (Tableaux 2 et 3 ). La famille des Fabaceae est la plus représentée (13 espèces soit 24,52\%). Elle est suivie de celles des Anacardiaceae, des Combretaceae et des Rutaceae qui ont chacune 4 espèces $(7,5 \%)$. Cette composition floristique varie en fonction des communes et est plus importante dans less communes de Kartiack et Mangagoulack qui ont enregistré 39 espèces réparties dans 36 et 35 genres et 17 et 2 familles respectivement. La commune de Mlomp est moins riche en espèces avec 27 espèces réparties en 26 genres et 14 familles.

\section{Analyse fréquentielle}

L'analyse du Tableau 4 montre que dans les parcs agroforestiers de l'arrondissement de Tendouck les espèces ligneuses les plus fréquentes sont: Guiera senegalensis $(60,2 \%)$, Cassia sieberiana $(51,02 \%)$ et Spondias mombin $(47,95 \%)$. Cependant, Guiera senegalensis, Parkia biglobosa, Terminalia macroptera, Combretum micranthum et Dialium guineense sont les plus fréquentes dans la commune de Diégoune avec des fréquences de présence respectives de $95,23 \% ; 85,71 \% ; 71,42 \%$ et $66,66 \%$. Dans la commune de Kartiack les espèces les plus fréquentes sont Cassia sieberiana $(68,18 \%)$ et Icacina senegalensis $(54,54 \%)$. Elles sont suivies par les espèces Dialium guineense, Faidherbia albida et Combretum micranthum avec chacune une fréquence de présence de 50\%. Dans la commune de Balinghore, ce sont les espèces Faidherbia albida et Anacardium occidentale qui sont les plus fréquentes avec respectivement $56,25 \%$ et $50 \%$ de fréquence de présence. Les espèces les plus fréquentes dans la commune de Mangagoulack sont Pterocarpus erinaceus (60\%), Cassia sieberiana $(56,66 \%)$ et Terminalia macroptera (53,33\%). Dans la commune de Mlomp, l'espèce Parkia biglobosa qui est présente dans tous les relevés est la plus fréquente (100\%). Elle est suivie des espèces Faidherbia albida et Piliostigma thonningii avec une fréquence de présence de $88,88 \%$ chacune puis des espèces Cassia sieberiana $(66,66 \%)$ et Borassus akeassii $(66,66 \%)$.

\section{Caractéristiques structurales de la végétation ligneuse des parcs}

Le taux de recouvrement moyen de la végétation ligneuse des parcs agroforestiers dans l'arrondissement est de 18,82\% (Tableau 5). Il est plus important à Mlomp $(27,65 \%)$ et plus faible à Mangagoulack (12,55\%). Les espèces qui ont les taux de recouvrement les plus élevés au niveau de l'arrondissement sont 
Parkia biglobosa (4,7\%), Dialium guineense $(2,6 \%)$ et Faidherbia albida (2,46\%).

Suivant les communes, les espèces ayant le taux de couverture le plus important sont: Parkia biglobosa $(9,14 \%)$ et Dialium guineense $(4,37 \%)$ pour Diégoune; Parkia biglobosa $(6,96 \%)$, et Dialium guineense $(5,9 \%)$ pour Kartiack; Khaya senegalensis $(3,44 \%)$ et Mangifera indica $(1,6 \%)$ pour Mangagoulack; Parkia biglobosa $(11,6 \%)$ et Faidherbia albida $(7,22 \%)$ pour Mlomp et enfin Faidherbia albida (2,6\%) et Parkia biglobosa $(2,05 \%)$ pour Balinghore.

La surface terrière moyenne est de 3,21 $\mathrm{m}^{2} /$ ha. Elle varie de $0,43 \mathrm{~m}^{2} /$ ha à Mlomp à 4,2 $\mathrm{m}^{2} /$ ha à Balinghore (Tableau 5). Les espèces dont les surfaces terrières sont les plus importantes au niveau de l'arrondissement sont: Parkia biglobosa (0,6 $\left.\mathrm{m}^{2} / \mathrm{ha}\right)$, Faidherbia albida $\left(0,46 \mathrm{~m}^{2} / \mathrm{ha}\right)$, et Dialium guineense $\left(0,28 \mathrm{~m}^{2} / \mathrm{ha}\right)$.

Suivant les communes, les espèces qui ont les surfaces terrières les plus importantes sont : Parkia biglobosa $\left(1,11 \mathrm{~m}^{2} / \mathrm{ha}\right)$ et Dialium guineense $\left(0,34 \mathrm{~m}^{2} / \mathrm{ha}\right)$ pour Diègoune ; Faidherbia albida $\left(0,63 \mathrm{~m}^{2} / \mathrm{ha}\right)$ et Parkia biglobosa $\left(0,61 \mathrm{~m}^{2} / \mathrm{ha}\right)$ pour Kartiack; Pterocarpus erinaceus $\left(0,37 \quad \mathrm{~m}^{2} / \mathrm{ha}\right)$ et Faidherbia albida $\left(0,29 \mathrm{~m}^{2} / \mathrm{ha}\right)$ pour Mangagoulack; Parkia biglobosa $\left(0,16 \mathrm{~m}^{2} / \mathrm{ha}\right)$ et Faidherbia albida $\left(0,14 \mathrm{~m}^{2} / \mathrm{ha}\right)$ pour Mlomp et enfin Faidherbia albida $\left(0,63 \mathrm{~m}^{2} / \mathrm{ha}\right)$, et Dialium guineense $\left(0,49 \mathrm{~m}^{2} / \mathrm{ha}\right)$ pour Balinghore.

La densité observée est en moyenne de 42 individus/ha dans les parcs (Tableau 5). Elle est plus importante dans la commune de Katiack avec 57 individus/ha et plus faible dans la commune de Diégoune avec 34 individus/ha. Les espèces qui ont les densités les plus élevées au niveau de l'arrondissement sont: Dialium guineense (6 pieds/ha), Parkia biglobosa (6 pieds/ha), Anacardium occidentale (5 pieds/ha) et Faidherbia albida (4 pieds/ha).

Considérant les communes, les espèces qui ont les plus grandes densités sont : Dialium guineense (9 pieds/ha), Parkia biglobosa (9 pieds/ha) pour Diégoune; Dialium guineense (12 pieds/ha) et Parkia biglobosa (8 pieds/ha) pour Kartiack; Pterocarpus erinaceus (5 pieds/ha) et Anacardium occidentale (5 pieds/ha) pour Mangagoulack; Parkia biglobosa (12 pieds/ha), et Faidherbia albida (12 pieds/ha) pour Mlomp et enfin Terminalia macroptera (10 pieds/ha), Faidherbia albida (4 pieds/ha) et Anacardium occidentale (4 pieds/ha) pour Balinghore.

Les parcs agroforestiers de l'arrondissement sont très diversifiés avec un Indice de Shannon de 3,91 et un indice de Pielou de 0,79 (Tableau 5). Considérant les communes, la diversité est plus importante dans la commune de Mangagoulack ( $\mathrm{H}=4,15$; $\mathrm{E}=0,78)$ et moins importante dans celle de Balinghore $(\mathrm{H}=2,88 ; \mathrm{E}=0,57)$.

Le taux de régénération du peuplement ligneux des parcs est de $88,19 \%$. Il est plus élevé à Kartiack $(94,5 \%)$ et moins important à Mangagoulack avec 80,9\% (Tableau 5).

\section{Structure de la végétation ligneuse des parcs Structure par classes de hauteur (ligneux de hauteur $>2 \mathrm{~m}$ )}

L'analyse de la Figure 3 montre que dans l'arrondissement de Tendouck, les individus de petite taille (hauteur comprise entre 2 et $4 \mathrm{~m}$ ) représentent à eux seuls 24,73\% des individus. La même tendance est observée à Diégoune (29,47\%), Kartiack (27,21\%), Mangagoulack (26,41\%) et Balinghore (20,91 \%). A Mlomp c'est la classe de hauteur [10-12 $\mathrm{m}$ [qui est la plus représentée avec $24,05 \%$ des individus du peuplement. Dans la commune de Mlomp, les individus de hauteur supérieure à $20 \mathrm{~m}$ ne sont pas représentés et sont très faiblement représentés dans celle de Diégoune $(1,73 \%)$. La structure par classes de hauteur est globalement équilibrée dans l'ensemble des communes de l'arrondissement.

\section{Structure par classes de diamètre (ligneux de diamètre $>5 \mathrm{~cm}$ )}

L'analyse de la Figure 4 révèle qu'aussi bien dans l'arrondissement de Tendouck de façon générale que dans les communes, les individus de diamètre compris entre 5 et $35 \mathrm{~cm}$ (deux premières classes) sont les mieux représentés exceptée la commune Mlomp. Ces deux premières classes regroupent à elles seules $75,18 \%$ des individus dans l'arrondissement de Tendouck tandis que dans 
les communes elles regroupent $81,15 \%$; $79,26 \% ; 74,55 \% ; 66 \%$ et $66,63 \%$ des individus respectivement à Kartiack, Mangagoulack, Diégoune, Balinghore et Mlomp. Les individus de diamètre supérieur à $50 \mathrm{~cm}$ sont faiblement représentés dans l'ensemble des communes de l'arrondissement. Dans la commune de Mlomp ce sont les individus de diamètre compris entre 30 et $35 \mathrm{~cm}$ qui sont les mieux représentés. Toutefois, le peuplement des parcs est équilibré dans l'ensemble des communes avec une structure en « $\mathrm{L}$ » caractéristique d'un peuplement jeune et stable.

\section{Typologie des parcs suivant les communes}

Les résultats de l'inertie et des valeurs propres de l'Analyse en Composantes Principales (ACP) sur les paramètres quantitatifs structuraux et floristiques selon les communes sont présentés dans le Tableau 6. Le plan factoriel constitué par ces deux axes F1 x F2 qui représentent $82,5 \%$ de l'inertie totale a permis une bonne représentation de l'information contenue dans la matrice.

L'analyse en composantes principales a permis de montrer que les indices de diversité sont positivement corrélés à l'axe des abscisses qui caractérise ainsi la diversité des parcs agroforestiers. Par contre l'axe F2 caractérise un gradient positif de la richesse spécifique, de la densité et de la régénération des parcs.

Ainsi, l'ACP a permis de distinguer les parcs du groupe A situés du côté des abscisses positives représentés par les parcs de la commune de Mangagoulack (A1) caractérisés par une diversité spécifique et une richesse spécifique élevées et les parcs de la commune de Mlomp (A2) caractérisés par une densité, une surface terrière, un taux de recouvrement et un taux de régénération faibles. Ces parcs s'opposent aux parcs du groupe $\mathrm{B}$ situés du côté des abscisses négatives qui se distinguent en deux sous-groupes (B1 et B2). Le sous-groupe B1 renferme les parcs de la commune de Kartiack caractérisés par une densité, une surface terrière, un taux de recouvrement et un taux de régénération élevés. Quant au sous-groupe B2, il regroupe les parcs des communes de Diégoune et Balinghore caractérisés par une diversité spécifique et richesse spécifique faibles (Figure $5)$.

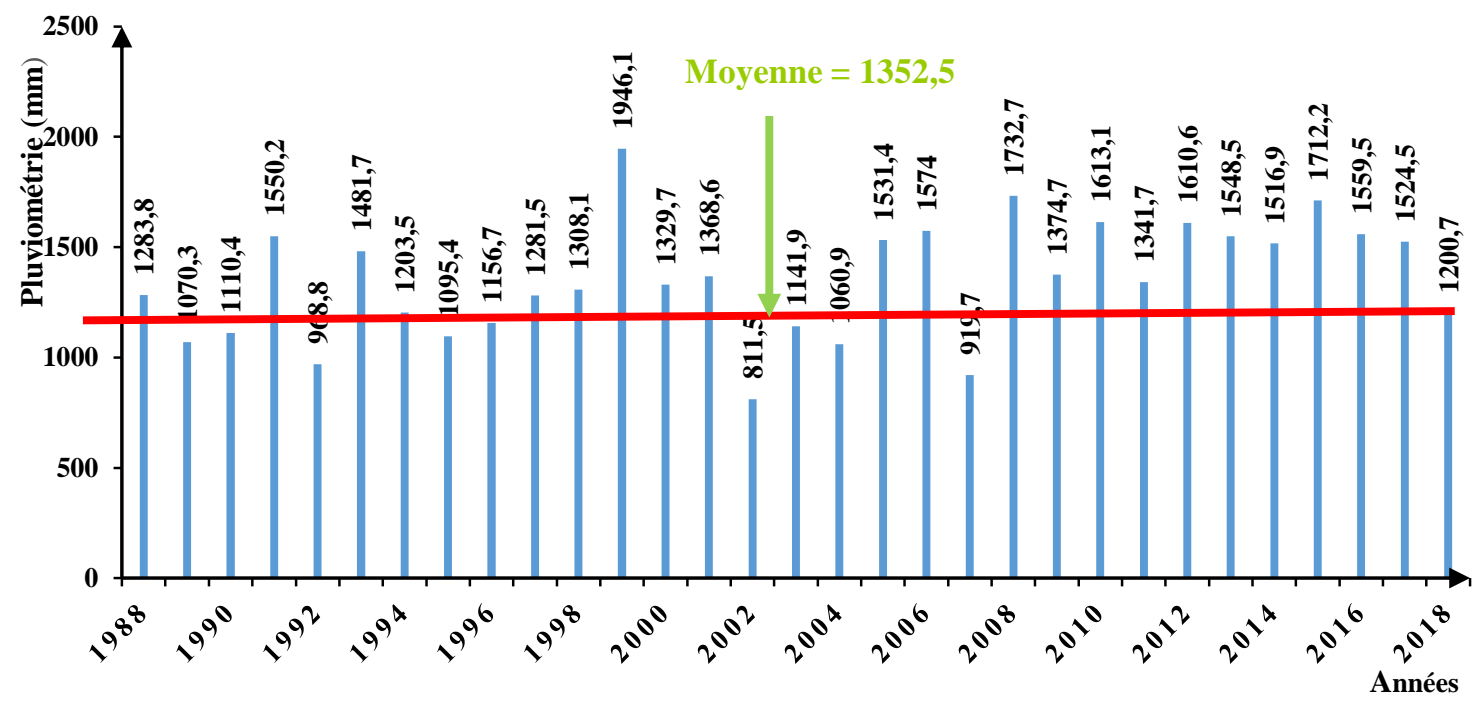

Figure 2: Variation de la pluviométrie moyenne annuelle de la région de Ziguinchor de 1988 à 2018 (ANACIM, 2019). 
Tableau 2 : Liste des espèces recensées dans l'arrondissement de Tendouck.

\begin{tabular}{|c|c|c|c|c|c|c|c|c|}
\hline \multirow{2}{*}{ Familles } & \multirow{2}{*}{ Genres } & \multirow{2}{*}{ Espèces } & \multicolumn{5}{|c|}{ Communes } & \multirow[t]{2}{*}{ Arr. Tendouck } \\
\hline & & & Manga goulack & Kartiack & Diegoune & Mlomp & Balinghore & \\
\hline \multirow{4}{*}{ Anacardiaceae } & Anacardium & Anacardium occidentale $\mathrm{L}$. & + & + & + & + & + & + \\
\hline & Lannea & Lannea acida $\mathrm{L}$. & - & - & - & + & - & + \\
\hline & Manguifera & Mangifera indica $\mathrm{L}$. & + & + & - & + & + & + \\
\hline & Spondias & Spondias mombin L. & - & - & - & + & - & + \\
\hline \multirow{3}{*}{ Annonaceae } & Annona & Annona glauca Schumach. \& Thonn. & + & + & + & + & - & 3 \\
\hline & Annona & Annona senegalensis Pers. & + & + & + & + & + & + \\
\hline & Uvaria & Uvaria chamea $(\mathrm{Rac})$ & + & + & + & - & + & + \\
\hline \multirow{3}{*}{ Apocynaceae } & Holarrhena & Holarrhena floribunda L. & - & + & + & - & + & + \\
\hline & Landolphia & Landolphia heudolotii A.DC. & + & + & + & + & - & + \\
\hline & Saba & Saba senegalensis (A.DC.) Pichon & + & + & + & + & + & + \\
\hline \multirow[t]{2}{*}{ Arecaceae } & Borassus & Borassus akeassii Bayton. Ouédr. \& Guinko. & + & + & + & + & + & + \\
\hline & Elaeis & Elaeis guineensis Jacq. & + & + & - & + & + & + \\
\hline Bignoniaceae & Markhamia & Markhamia tomentosa (Benth.) & + & - & + & + & - & + \\
\hline \multirow[t]{2}{*}{ Bombacaceae } & Adonsonia & Adonsonia digitata $\mathrm{L}$. & - & - & - & - & + & + \\
\hline & Neobouldia & Newbouldia lewis (P.Beauv.) Seem. & - & + & - & - & - & + \\
\hline \multirow{2}{*}{ Chrysobalanaceae } & Ceiba & Ceiba pentandra (L.) Gaertn. & + & - & + & + & + & + \\
\hline & Neocaria & Neocarya macrophylla (Sabine) Prance & - & + & + & - & + & + \\
\hline \multirow{5}{*}{ Combretaceae } & Combretum & Combretum glutinosum Perr. Ex DC. & + & - & - & - & + & + \\
\hline & & Combretum micranthum G.Don & + & + & + & + & - & + \\
\hline & Guera & Guiera senegalensis J.F. Gmel & + & + & + & + & + & + \\
\hline & Parinari & Parinari excelsa Sabine. & + & + & - & - & - & + \\
\hline & Terminalia & Terminalia macroptera Guill.\& Perr. & + & + & + & - & + & + \\
\hline \multirow{4}{*}{ Fabaceae } & Acacia & Aacia holosericea A.Cunn. ex G.Don & + & - & - & - & - & + \\
\hline & Albizia & Albizia adianthifolia W.Wight & + & - & - & - & - & + \\
\hline & Cassia & Cassia sieberiana DC. & + & + & + & + & + & + \\
\hline & Daniela & Daniellia oliveri Benn. & + & + & + & - & - & + \\
\hline
\end{tabular}


I. COLY et al. / Int. J. Biol. Chem. Sci. 14(5): 1558-1575, 2020

\begin{tabular}{|c|c|c|c|c|c|c|c|c|}
\hline & Detarium & Detarium senegalenseJ.F.Gmel. & + & + & + & + & - & + \\
\hline & Dialium & Dialium guineense Willd & + & + & + & + & + & + \\
\hline & Dicrostachys & Dicrostachys cineria (L.) Wight. \& Arn. & + & + & + & + & + & + \\
\hline & Erithrina & Erythrina senegalensis L. & + & + & + & + & + & + \\
\hline & Faidherbia & Faidherbia albida( Delile) A.Chev. & + & + & + & + & + & + \\
\hline & Parkia & Parkia biglobosa (Jacq.) R.Br. Ex G.Don & + & + & + & + & + & + \\
\hline & Pliostigma & Piliostigma thonningii (Schum.) Milne-Redh. & + & + & + & + & - & + \\
\hline & Prosopis & Prosopis africana (Guill. \& Perr.) Taub. & - & + & + & + & + & + \\
\hline & Pterocarpus & Pterocarpus erinaceus Poir. & + & + & + & - & + & + \\
\hline Icacinaceae & Icacina & Icacina senegalensis Juss. & + & + & + & + & + & + \\
\hline Lamiaceae & Vitex & Vitex doniana Sweet & + & - & + & - & - & + \\
\hline \multirow{2}{*}{ Malvaceae } & Bombax & Bombax costatum Pellegr. \& Vuillet & - & + & - & - & - & + \\
\hline & Cola & Cola cordifolia (Cav.) R. Br & - & + & - & - & + & + \\
\hline \multirow{2}{*}{ Meliaceae } & Azadirachta & Azadirachta indica A.Juss. & + & + & - & + & + & + \\
\hline & khaya & khaya senegalensis (Desr.) A.Juss. & + & + & + & - & + & + \\
\hline \multirow{3}{*}{ Moracea } & Ficus & Ficus asperifolia & - & + & - & - & + & + \\
\hline & Ficus & Ficus sycomorus L. & - & + & - & - & + & + \\
\hline & Ficus & Ficus exasperata $\mathrm{Vahl}$ & + & + & - & - & + & + \\
\hline Polygalaceae & Securidaca & Securidaca longepedunculata Fres. & - & - & + & - & - & + \\
\hline Rubiaceae & Gardenia & Gardenia ternifolia J.Ellis & - & + & - & - & - & + \\
\hline \multirow{4}{*}{ Rutaceae } & Citrus & Citrus lemon(L.) Burm. F. & + & - & - & - & + & + \\
\hline & Citrus & Citrus reticulata $\mathrm{L}$. & + & - & - & - & - & + \\
\hline & Citrus & Citrus sinensis (L.) Osbeck & + & - & - & - & - & + \\
\hline & Fagara & Fagara zantholoxyloides Lam. & - & + & - & + & - & + \\
\hline Sapindaceae & Allophylus & Allophylus africanus P.Beauv. & + & + & + & - & + & + \\
\hline Simaroubaceae & Hunnoa & Hannoa undulata (Guill.\& Perr) Planch. & + & + & + & + & + & + \\
\hline Ulmaceae & Celtis & Celtis integrifolia Lam. & - & - & + & - & + & + \\
\hline Total & 45 & 53 & 38 & 39 & 32 & 27 & 33 & 53 \\
\hline
\end{tabular}


Tableau 3 : Effectif des taxons dans les différentes communes de l'arrondissement de Tendouck.

\begin{tabular}{ccccccc}
\cline { 2 - 7 } & & \multicolumn{3}{c}{ Communes } & & $\begin{array}{c}\text { Arrondissement } \\
\text { Tendouck }\end{array}$ \\
\hline Taxons & Diégoune & Kartiack & Mangagoulack & Balinghore & Mlomp & \\
\hline Familles & 17 & 17 & 20 & 16 & 14 & 20 \\
Genres & 29 & 36 & 35 & 30 & 26 & 45 \\
Espèces & 32 & 39 & 39 & 32 & 27 & 53 \\
\hline
\end{tabular}

Tableau 4: Fréquences de présence des espèces ligneuses dans les parcs agroforestiers des communes de l'arrondissement de Tendouck.

\begin{tabular}{|c|c|c|c|c|c|c|}
\hline Zones Espèces & Diégoune & Kartiack & Balinghore & Mangagoulack & Mlomp & $\begin{array}{c}\text { Arrondisse } \\
\text { ment }\end{array}$ \\
\hline $\begin{array}{l}\text { Acacia holosericea A.Cunn. } \\
\text { ex G.Don }\end{array}$ & 0 & 0 & 0 & 3,33 & 0 & 1,02 \\
\hline Adansonia digitata $\mathrm{L}$. & 0 & 0 & 6,25 & 0 & 0 & 1,02 \\
\hline Albizia adiantifolia W.Wight & 0 & 0 & 0 & 16,66 & 0 & 5,1 \\
\hline Allophylus africanus P.Beauv. & 0 & 9,09 & 0 & 6,66 & 0 & 4,08 \\
\hline Anacardium occidentale L. & 33,33 & 40,9 & 50 & 46,66 & 44,44 & 42,85 \\
\hline $\begin{array}{l}\text { Annona glauca Schumach. \& } \\
\text { Thonn. }\end{array}$ & 0 & 13,63 & 0 & 3,33 & 11,11 & 5,1 \\
\hline Annona senegalensis Pers. & 33,33 & 31,81 & 25 & 36,66 & 44,44 & 33,67 \\
\hline Azadirachta indica A.Juss. & 0 & 0 & 6,25 & 23,33 & 11,11 & 9,18 \\
\hline $\begin{array}{l}\text { Bombax costatum Pellegr. \& } \\
\text { Vuillet }\end{array}$ & 0 & 9,09 & 0 & 0 & 0 & 2,04 \\
\hline $\begin{array}{l}\text { Borassus akeassii Bayton. Ou. } \\
\text { \& Guinko. }\end{array}$ & 38,09 & 31,81 & 43,75 & 10 & 66,66 & 31,63 \\
\hline Cassia sieberiana DC. & 47,61 & 68,18 & 12,5 & 56,66 & 66,66 & 51,02 \\
\hline Ceiba pentandra (L.) Gaertn. & 9,52 & 0 & 6,25 & 6,66 & 22,22 & 7,14 \\
\hline Celtis toka Forssk. & 0 & 0 & 6,25 & 0 & 0 & 1,02 \\
\hline Citrus lemon (L.) Burm. F. & 0 & 0 & 6,25 & 6,66 & 0 & 3,06 \\
\hline Citrus reticulata $\mathrm{L}$. & 0 & 0 & 0 & 3,33 & 0 & 3,06 \\
\hline Citrus sinensis (L.) Osbeck & 0 & 0 & 0 & 16,66 & 0 & 5,1 \\
\hline Cola cordifolia (Cav.) R. Br. & 0 & 9,09 & 0 & 0 & 11,11 & 3,06 \\
\hline $\begin{array}{l}\text { Combretum glutinosum Perr. } \\
\text { Ex DC. }\end{array}$ & 0 & 0 & 0 & 3,33 & $\mathbf{5 5 , 5 5}$ & 1.02 \\
\hline Combretum micrantum G.Don & 71,42 & $\mathbf{5 0}$ & 0 & 40 & 0 & 43,87 \\
\hline Daniellia oliveri Benn. & 42,85 & 4,54 & 0 & 13,33 & 0 & 14,28 \\
\hline $\begin{array}{l}\text { Detarium senegalense } \\
\text { J.F.Gmel. }\end{array}$ & 14,28 & 13,63 & 0 & 33,33 & 33,33 & 12,24 \\
\hline Dialium guineense Willd & 66,66 & 50 & 18,75 & 50 & 22,22 & 43,87 \\
\hline $\begin{array}{l}\text { Dicrostachys cinerea (L.) } \\
\text { Wight. \& Arn. }\end{array}$ & 47,61 & 13,63 & 31,25 & 36,66 & 22,22 & 28,57 \\
\hline Elaeis guineensis Jacq. & 0 & 13,63 & 12,5 & 10 & 22,22 & 10,20 \\
\hline Erythrina senegalensis L. & 9,52 & 27,27 & 6,25 & 46,66 & 11,11 & 22,44 \\
\hline Fagara zanthoxyloides Lam. & 0 & 4,54 & 0 & 3,33 & 0 & 2,04 \\
\hline $\begin{array}{l}\text { Faidherbia albida ( Delile) } \\
\text { A.Chev. }\end{array}$ & 9,52 & $\mathbf{5 0}$ & 56,25 & 20 & $\mathbf{8 8 , 8 8}$ & 36,73 \\
\hline Ficus asperifolia & 0 & 0 & 12,5 & 0 & 0 & 2,04 \\
\hline \multicolumn{7}{|c|}{1567} \\
\hline
\end{tabular}


Ficus sycomorus $\mathrm{L}$.

Ficus exasperata Vahl

Gardenia ternifolia J.Ellis

Guiera senegalensis J.F. Gmel

Hunnoa undulata (Guill.\&

Perr) Planch.

Icacina senegalensis Juss.

Uvaria chamea (Rac)

khaya senegalensis (Desr.)

A.Juss.

Landolphia heudolotii A.DC.

Lannea acida $\mathrm{L}$.

Mangifera indica $\mathrm{L}$.

Markhamia tomentosa

(Benth.)

Newbouldia laevis (P.Beauv.)

Seem.

Neocarya macrophylla

(Sabine) Prance

Holarrhena floribunda L.

Parinari excelsa Sabine.

Parkia biglobosa (Jacq.) R.Br.

Ex G.Don

Piliostigma thonningii

(Schum.) Milne-Re.

Prosopis africana (Guill. \&

Perr.) Taub.

Pterocarpus erinaceus Poir.

Saba senegalensis (A.DC.)

Pichon

Securidaca longipedunculata

Fres.

Spondias mombin L.

Terminalia macroptera

Guill.\& Perr.

Vitex doniana Sweet

\begin{tabular}{|c|c|c|c|c|c|}
\hline 0 & 4,54 & 6,25 & 0 & 0 & 2,04 \\
\hline 0 & 4,54 & 12,5 & 6,66 & 0 & 5,1 \\
\hline 0 & 4,54 & 0 & 0 & 0 & 1,02 \\
\hline 95,23 & 40,9 & 50 & 60 & 44,44 & 60,2 \\
\hline 42,85 & 40,9 & 18,75 & 6,66 & 22,22 & 25,51 \\
\hline 28,57 & 54,54 & 31,25 & 16,66 & 22,22 & 35,71 \\
\hline 23,8 & 9,09 & 12,5 & 6,66 & 77,77 & 11,22 \\
\hline 9,52 & 45,45 & 12,5 & 50 & 0 & 29,59 \\
\hline 14,28 & 4,54 & 0 & 53,33 & 0 & 20,4 \\
\hline 4,76 & 9,09 & 0 & 0 & 0 & 12,24 \\
\hline 0 & 0 & 6,25 & 20 & 0 & 7,14 \\
\hline 23,8 & 9,09 & 0 & 3,33 & 33,33 & 2,04 \\
\hline 0 & 13,63 & 0 & 0 & 11,11 & 8,16 \\
\hline 0 & 9,09 & 31,25 & 0 & 0 & 11,22 \\
\hline 38,09 & 0 & 6,25 & 0 & 0 & 2,04 \\
\hline 0 & 40,9 & 0 & 3,33 & 11,11 & 43,87 \\
\hline 85,71 & 27,27 & 31,25 & 6,66 & 100 & 31,63 \\
\hline 38,09 & 18,18 & 0 & 30 & 88,88 & 12,24 \\
\hline 14,28 & 27,27 & 18,75 & 0 & 22,22 & 32,65 \\
\hline 38,09 & 13,63 & 12,25 & 60 & 0 & 15,3 \\
\hline 9,52 & 27,72 & 18,75 & 23,33 & 11,11 & 3,06 \\
\hline 14,28 & 0 & 0 & 0 & 0 & 1,02 \\
\hline 0 & 31,81 & 0 & 0 & 11,11 & 47,95 \\
\hline 76,19 & 0 & 50 & 53,33 & 0 & 7,14 \\
\hline 9,52 & 0 & 0 & 16,66 & 0 & 1,02 \\
\hline
\end{tabular}

Tableau 5 : Paramètres structuraux de la végétation ligneuse des parcs agroforestiers des communes de l'arrondissement de Tendouck.

\begin{tabular}{|c|c|c|c|c|c|c|}
\hline & & & & & & \multirow{3}{*}{ Arrondissemen } \\
\hline \multirow{2}{*}{ Paramètres } & \multicolumn{5}{|c|}{ Communes } & \\
\hline & Diég. & Kart. & Baling. & Manga. & Mlomp & \\
\hline Taux recouvrement $(\%)$ & 20,17 & 25,53 & 14,62 & 12,55 & 27,65 & 18,82 \\
\hline Surface terrière $\left(\mathrm{m}^{2} / \mathrm{ha}\right)$ & 2,6 & 3,51 & 4,2 & 2,54 & 0,43 & 3,21 \\
\hline Densité (ind./ha) & 34,47 & 57,27 & 37 & 37,46 & 41,33 & 41,55 \\
\hline Taux de régénération & 87,79 & 94,54 & 82,58 & 80,9 & 85,28 & 88,19 \\
\hline Diversité $\quad \mathrm{H}$ & 2,99 & 3,57 & 2,88 & 4,15 & 4,49 & 3,91 \\
\hline spécitique & 0,59 & 0,67 & 0,57 & 0,78 & 0,7 & 0,79 \\
\hline
\end{tabular}

Dieg $=$ Diegoune $;$ Kart $=$ Kartiack $;$ Baling $=$ Balinghore $;$ Manga $=$ Mangagoulack 

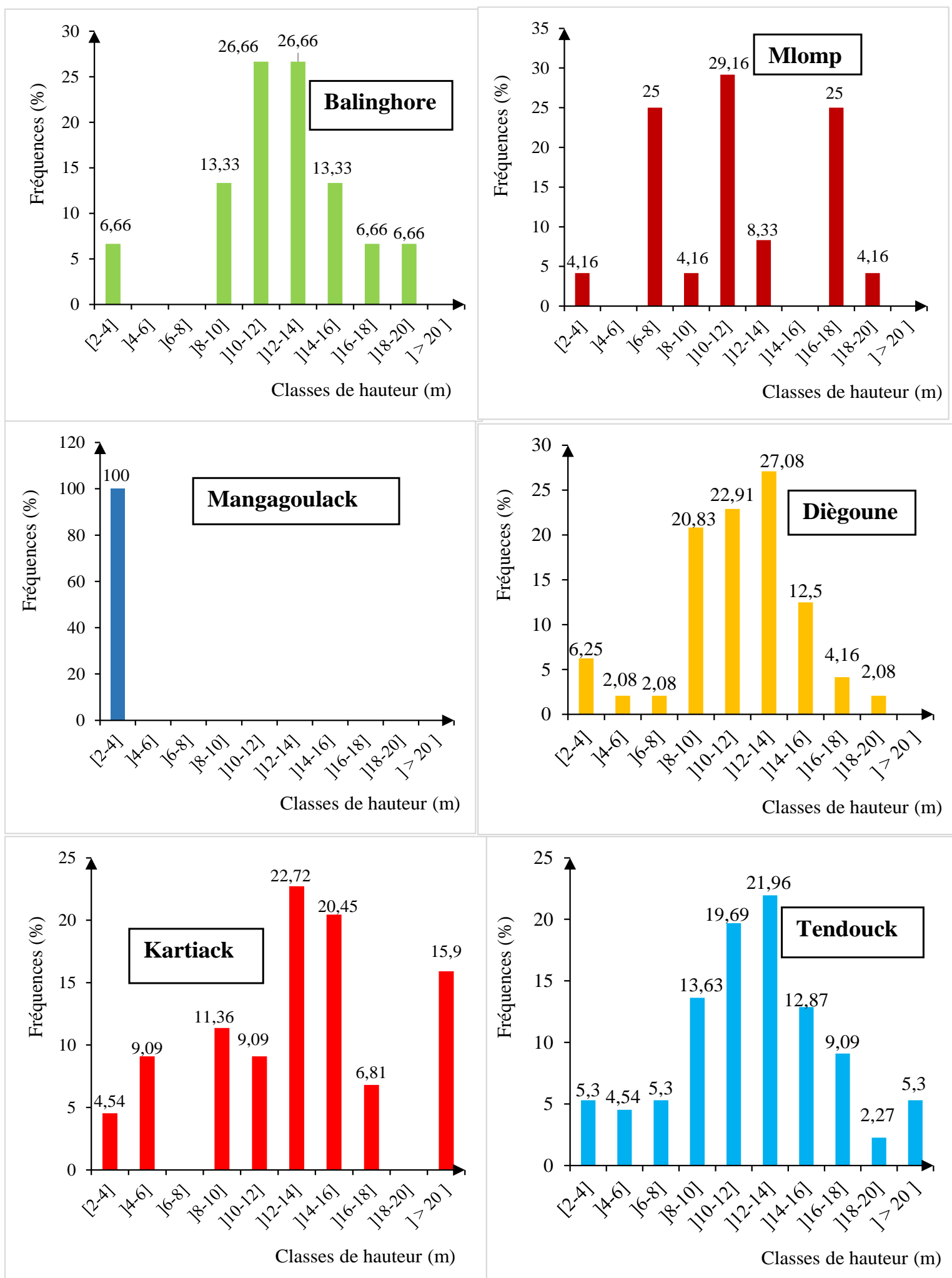

Figure 3 : distribution des individus du peuplement ligneux des parcs agroforestiers par classes de hauteur dans les communes et l'arrondissement de Tendouck. 


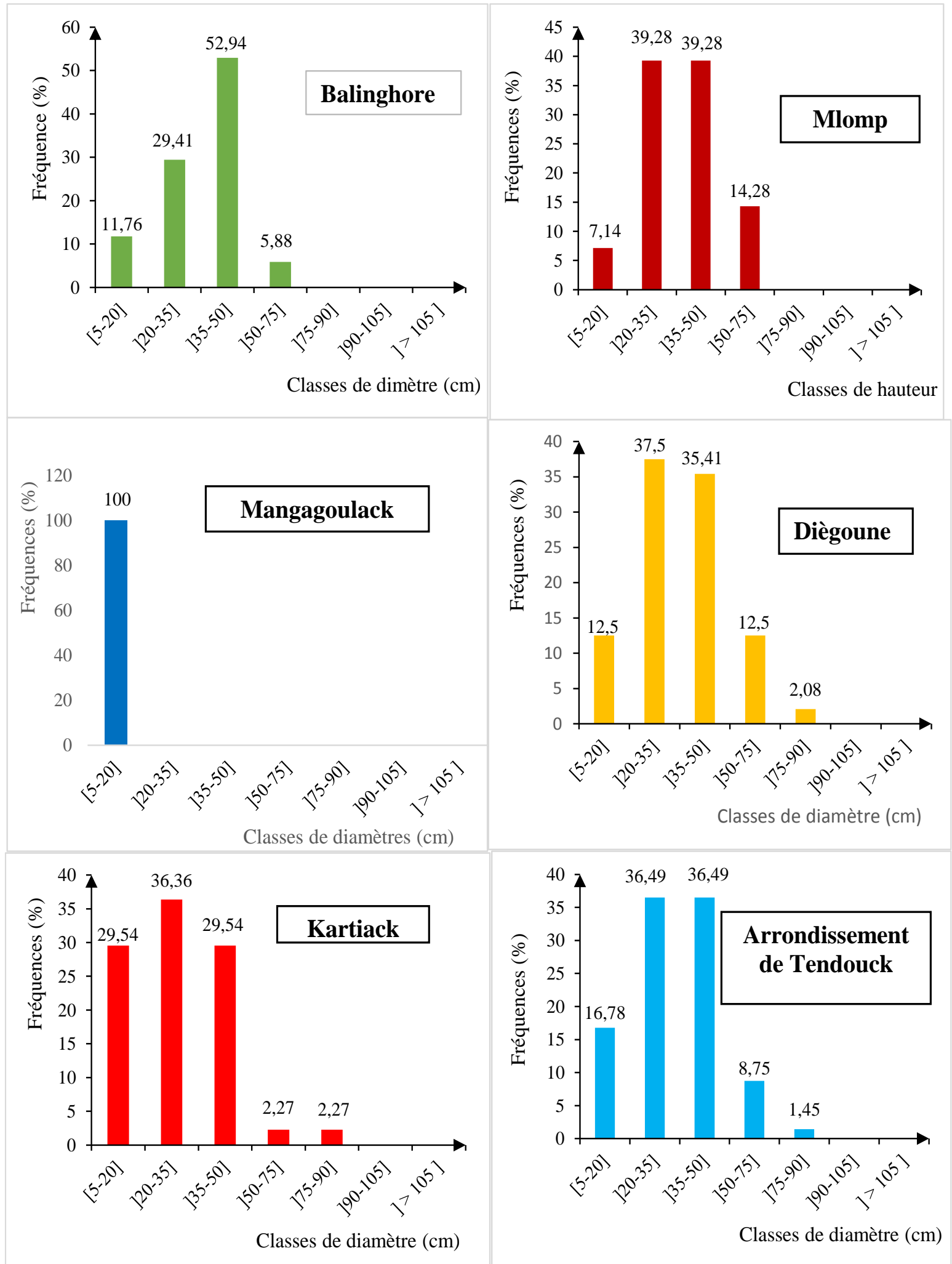

Figure 4: distribution des individus du peuplement ligneux des parcs agroforestiers par classes de diamètre dans les communes et l'arrondissement de Tendouck. 
Tableau 6 : valeur propres et inerties des premiers axes de l'ACP.

\begin{tabular}{ccccc}
\hline & F1 & F2 & F3 & F4 \\
\hline Valeurs propres & 3,988 & 2,593 & 1,239 & 0,180 \\
inerties (\%) & 49,852 & 32,414 & 15,490 & 2,245 \\
Inerties Cumulées (\%) & 49,852 & 82,265 & 97,755 & 100,000 \\
\hline
\end{tabular}

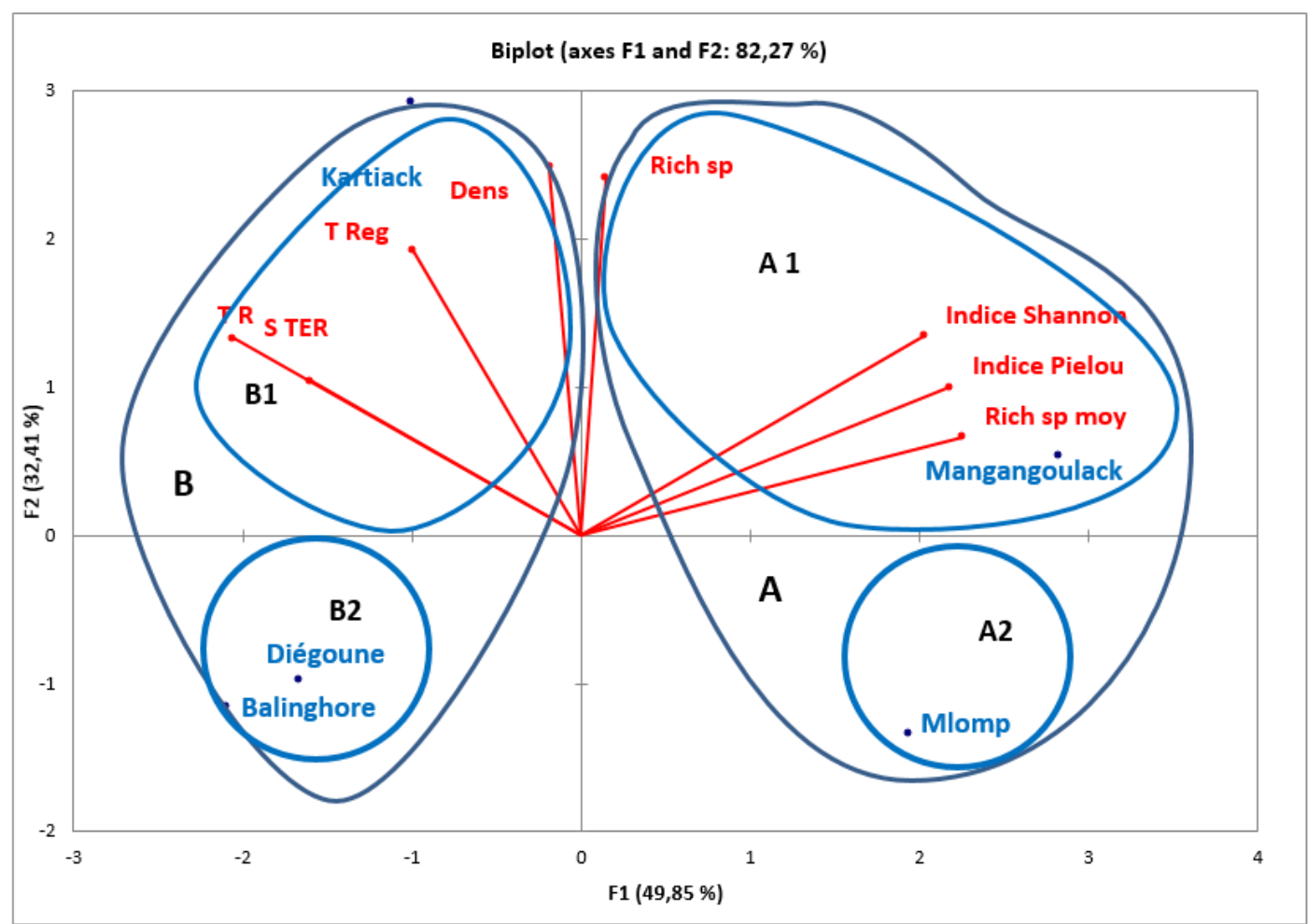

$\mathrm{T} \mathrm{R}=$ taux de recouvrement ; Dens= densité $; \mathrm{S} \mathrm{T}=$ surface terrière ; $\mathrm{T} \mathrm{Reg}=$ taux de régénération ; rich $\mathrm{sp}=$ richesse spécifique.

Figure 5 : Types de parcs agroforestiers définis par l'ACP des 5 communes x 8 variables (paramètres structuraux et floristiques des parcs de l'arrondissement de Tendouck).

\section{DISCUSSION}

L'étude de la végétation ligneuse des parcs agroforestiers de l'arrondissement de Tendouck a révélé la présence de 53 espèces réparties dans 45 genres et 20 familles. Ces résultats sont proches de ceux de Ngom et al. (2018) qui ont obtenu au niveau des parcs agroforestiers à Elaeis guineensis en Basse Casamance 51 espèces à Karounate, 47 espèces à Kabiline et 50 espèces à Kaguite. Ils sont aussi proches de ceux de Diedhiou et al., (2014) qui ont trouvé 54 espèces 43 genres et 24 familles au niveau des parcs agroforestiers du terroir insulaire de Mar Fafaco dans la région de Fatick en zone soudano-sahélienne du Sénégal. Cependant la richesse spécifique est plus élevée comparée à celle trouvée par Coly et al. (2005) qui est de 35 espèces au 
niveau des champs du bassin versant de la Nema, dans le Niombato au Saloum. La présence de Guiera senegalensis sur $60 \%$ des relevés renseigne sur la capacité de cette espèce à s'adapter et à se multiplier rapidement dans les zones soudaniennes. Ce résultat corrobore celui de Mbow et al. (2005) qui ont trouvé au niveau des systèmes d'utilisation des terres du Sud-Ouest du Bassin arachidier que Guiera senegalensis avec une fréquence de présence de $59,7 \%$ y est plus fréquente que les autres espèces.

Les parcs agroforestiers de l'arrondissement de Tendouck sont très diversifiés en espèces. Pour un total de 53 espèces, 39 sont représentées à Mangagoulack et Karthiack, 32 à Diègoune et Balinghore et 27 à Mlomp. La diversité spécifique des parcs est très élevée avec un indice de Shannon de 3,9 bits dans l'arrondissement, 4,15 bits à Mangagoulack, 3,5 bits à Karthiack, 3,49 bits à Mlomp, 2,99 bits à Diègoune et 2,88 bits à Balinghore. Ces résultats corroborent aussi ceux de Ngom et al. (2018) qui ont obtenu des indices de Shannon élevés variant de 4,12 bits à 2,92 bits dans ces mêmes parcs à Elaeis guinennsis. Les indices d'équitabilité de Pielou obtenus dans l'arrondissement et dans les communes sont tous au-dessus de 0,5. Ils varient entre 0,79 (arrondissement) et 0,57 (Balinghore). Ceci pourrait s'expliquer par une conservation de la plupart des espèces présentes dans les champs par les populations du fait de leur intérêt socio-économique (Ndiaye, et al. 2017).

Les valeurs obtenues pour la densité (41,5 pieds/ha), la surface terrières $(3,21$ $\mathrm{m}^{2} /$ ha) et le taux de couverture $(18,82 \%)$ sont proches de celles trouvées par Natta et al. (2012) au niveau des parcs agroforestiers du nord Bénin à Tchallinga pour les deux premiers paramètres avec 45 arbres/ha pour la densité et $4,1 \mathrm{~m}^{2} /$ ha pour la surface terrière. Par contre pour nos résultats sont supérieurs à ceux obtenus par Yameogo et al. (2019) pour la densité. Ces derniers ont obtenu 8,25 pieds/ha dans les parcs agroforestiers de Vipalogo en zone nord soudanienne du Burkina Fasa.

L'analyse de la structure du peuplement des ligneux des parcs agroforestiers de l'arrondissement de Tendouck révèle un peuplement relativement jeune avec $24,73 \%$ des individus ayant une hauteur appartenant à la classe [2-4 m]. La structure par classes de hauteur est globalement équilibrée dans l'ensemble des communes de l'arrondissement. Dans l'arrondissement comme dans les communes les individus de diamètre compris entre 5 et $35 \mathrm{~cm}$ (deux premières classes) sont les mieux représentés excepté la commune Mlomp. Ces deux premières classes regroupent à elles seules $75,18 \%$ des individus dans l'arrondissement de Tendouck. Et dans les communes elles regroupent $81,15 \% ; 79,26 \% ; 74,55 \% ; 66 \%$ et $66,63 \%$ des individus respectivement à Kartiack, Mangagoulack, Diégoune, Balinghore et Mlomp. Les individus de diamètre supérieur à $50 \mathrm{~cm}$ sont faiblement représentés dans l'ensemble des communes de l'arrondissement. Ceci pourrait être lié à l'intervention humaine (Floquet, 2011). En effet, les agricultuers pour réduire la compétition des ligneux avec les cultures, coupent certains arbres de gros diamètres dans les champs. Toutefois le peuplement des parcs est équilibré dans l'ensemble des communes avec une structure en « $\mathrm{L}$ » caractéristique d'un peuplement jeune. Le taux de régénération du peuplement végétal est très élevé $(88,19 \%)$ dans l'arrondissement et au niveau des communes également (entre 94,54\% et $80,09 \%$ ). Ceci confirme les résultats de Guimbo et al. (2010) qui ont trouvé que le peuplement des parcs à Neocarya macrophylla et à Vitellaria paradoxa dans le sud-ouest nigérien se caractérise par une densité de régénération très élevée. 


\section{Conclusion}

Cette étude a permis de déterminer les caractéristiques de la végétation ligneuse des parcs agroforestiers dans l'arrondissement de Tendouck. La flore de la végétation ligneuse de ces parcs est riche de 53 espèces réparties en 45 genres et 20 familles. Les familles les plus représentées sont les Fabaceae (24,52\%), les Anacardiaceae (7,5\%), les combretaceae (7,5\%), les Rutaceae (7,5\%). L'espèce la plus fréquente dans les champs de l'arrondissement est Guiera senegalensis. La végétation ligneuse des parcs a une densité de 41,55 pieds à l'hectare, un taux de recouvrement de $18,82 \%$ et une surface terrière de $3,16 \mathrm{~m}^{2} / \mathrm{ha}$. Les indices de Shannon élevés dans les différentes communes et dans l'arrondissement en général montrent une diversité importante de la végétation ligneuse des parcs. Il apparait ainsi judicieux de poursuivre cette étude dans les autres départements de la Basse Casamance en vue de disposer d'une base de données indispensable pour une gestion durable des parcs agroforestiers de cette zone écogéographique.

\section{CONFLIT D'INTERETS}

Les auteurs déclarent quils n'ont pas de conflit dintérêts pour ce manuscrit.

\section{CONTRIBUTIONS DES AUTEURS}

IC : conduite de l'étude sur le terrain, collecte et traitement des données et rédaction du manuscrit. TCD : collecte et traitement des données. DN : Encadrement et correction du manuscrit. AB : collecte des données. OG : collecte des données.

\section{REMERCIEMENTS}

Nous remercions les agriculteurs pour leur accompagnement sur le terrain. Mais aussi Messieurs Arfang Ousmane Kémo GOUDIABY et Mamady DRAME pour leur participation active à l'inventaire de la végétation ligneuse.

\section{REFERENCES}

Badiane M, Camara B, Ngom D, Diedhiou M A A. 2019. Perception communautaire des parcs agroforestiers traditionnels à Faidherbia albida (Del.) Chev. En Basse Casamance, Sénégal. Afrique Science, 15(1): 214-226.

Bassène $\mathrm{C}$, Mbaye MS, Camara AB, Kane A, Gueye M, Sylla SN, Sambou B, Noba K. 2014. La flore des systèmes agropastoraux de la Basse Casamance (Sénégal) : cas de la communauté rurale de Mlomp. International Journal of Biological and Chemical, 8(5) Sciences.March 2015.17p. DOI: 10.4314/ijbcs. v8i5.28.

Berhaut J. 1967. Flore de Sénégal (2ème édition) clair Afrique: Dakar, Sénégal. 485p.

Boffa JM. 2000. Les parcs agroforestiers en Afrique de l'Ouest: clés de la conservation et d'une gestion durable. Unasylva 200, 51: 11-17.

Coly I, Akpo LE, Sarr D, Malou R, Dacosta H, Diom F. 2005. Caractérisation agroécologique du terroir de Néma en zone soudano-sahélienne au Sénégal: typologie des parcs agroforestiers. Agronomie Africaine, 17(1), AISA d'Abidjan : 53-62.

Goudiaby M. 2013. Les parcs agroforestiers en Basse Casamance : contribution de Parkia biglobosa (néré) à la réduction des risques de pauvreté des ménages de la communauté rurale de Mangangoulack, au Sénégal. Mémoire de maîtrise en agroforesterie. Université de Laval, Québec Canada.118p.

Diedhiou MAA, Faye E, Ngom D, Toure MA. 2014. Identification et caractérisation floristiques des parcs agroforestiers du terroir insulaire de Mar Fafaco (Fatick, Sénégal). Journal of Applied Biosciences, 79: 6855-6866.

DOI: 10.4314/jab.v79i1.11 
Floquet A. 2011. Typologie des systèmes agroforestiers au Bénin. In Atlas de la Biodiversité de l'Afrique de l'Ouest, (Volume 1), Sinsin B, Kampmann D. (eds). BIOTA. Cotonou \& Frankfurt/Main, Bénin. 725p

Guimbo ID, Mahamane A, Ambouta KJM. 2010. Peuplement des parcs à Neocarya macrophylla (Sabine) Prance et à Vitellaria paradoxa (Gaertn. C.F.) dans le sud-ouest nigérien : diversité, structure et régénération. International Journal of Biological and Chemical Sciences, 4(5): 1706-1720.

DOI: 10.4314/ijbcs.v4i5.65568

Kourouma K, Dissous FE, Ganglo, JC. 2013. Caractérisation écologique et structurale des parcs à Néré (Parkia biglobosa (Jacq.) R.Br.Ex.G.Don) du département de Donga au Nord-Ouest du Bénin. International Journal of Biological and Chemical Sciences, 7(2) : 726-738. DOI: 10.4314/ijbcs.v7i2.27

Labat JN. 1995. Végétation du nord-ouest du Michoacan Mexique. Instituto de Ecologia A. C.; 401p.

Lebrun J, Stork A. 1997. Enumération des Plantes à Fleurs d'Afrique Tropicale (Vol. I, II, III, IV). Conservatoire du Jardin Botanique de Genève ; 249, 341 et $712 \mathrm{p}$.

Mané A. 2007. L'importance de la route dans le développement socioéconomique d'une région : exemple de la départementale 200 ou boucle du Blouf. Université Cheikh Anta Diop de Dakar - Certificat d'aptitude à l'enseignement moyen (CAEM). 97p.

Mbow MA, Faye EH, L'aire M, Akpo LE, Diouf M. 2005. Diversité d'une végétation ligneuse soudanienne dans les systèmes d'utilisation des terres de SUDOUEST du bassin arachidier (Sénégal). Journal des Sciences et Technologies, 7: $21-34$.
Natta AK, Bachabi SF, Wallis NZ, Dicko A. 2012. Typologie et structure des parcs agroforestiers dans la zone soudanienne $\mathrm{du}$ nord bénin. Annales des Sciences Agronomiques, 16(1) : 67-90.

Ndiaye S, Charahabil M M, Ndiaye O, Diatta, M. 2017. Influence de la flore ligneuse associée dans la production des parcs à Anacarduim occidentale L. dans la communauté rurale de Djbanar (Casamance Sénégal). International Journal of Biological and Chemical Sciences, 11(2): $17 \mathrm{p} . \quad$ DOI: 10.4314/ijbcs.v11i2.5

Ngom D, Fall T, Sarr O, Diatta S, Akpo L E. 2013. Caractéristiques écologiques du peuplement ligneux de la réserve de biosphère du Ferlo (Nord Sénégal). Journal of Applied Biosciences, 65: 5008-5023.

DOI: 10.4314/jab.v65i0.89644

Ngom D, Camara B, Gomis Z. D, Sagna B. 2018. Cortège floristique, paramètres structuraux et indicateurs d'anthropisation des parcs agroforestiers à Elaeis guineensis Jacq. en Basse Casamance, Sénégal. Journal of Animal and Plant Sciences, 36(3) : 5919-5932. http://www.m.elewa.org/JAPS.

Ramade F. 2009. Eléments d'Écologie : Écologie Fondamentale (3ème édition). Dunod : Paris; 690p.

Roberts-Pichette P, Gillespie L. 1999. Protocole de Suivi de la Biodiversité Végétale Terrestre. Dans la section publication du site du réseau d'évaluation et de surveillance écologique (RESE) collection des publications hors-série du RESE Rapport $n^{\circ}$. Bureau de la coordination du RESE. Centre canadien des eaux intérieures. $138 \mathrm{p}$.

Sagna P. 2005. Dynamique du Climat et son évolution récente dans la partie Ouest de l'afrique occidentale. Thèse de Doctorat. Université Cheikh Anta Diop de 
Dakar.786 p.

Sané T. 2017. Vulnérabilité et adaptabilité des systèmes agraires à la variabilité climatique et aux changements sociaux en Basse Casamance (SUD-OUEST du Sénégal. Thèse de doctorat de Géographie et Environnement. UCAD, Université Sorbone, Paris cité. 377p.

Shannon C. E, Weaver W. 1949. The Mathematical Theory of Communication. Urbana, IL: The University of Illinois Press, 1-117.

Smektala G, Peltier R, Sibelet N, Leroy M, Manlay R, Njiti C F, Ntoupka M, Njiemoun A, Palo O. 2005. Parcs agroforestiers saheliens: de la
Conservation à l'Aménagement. VertigO, 6(2): $1-13$. https://doi.org/10.4000/vertigo.4410

Zoundi SJ, Léonidas H. 2003. Défis de l'accès des exploitations familiales aux innovations agricoles en Afrique de l'Ouest. Club du Sahel et de L'Afrique de l'Ouest. OCDE, Paris.15p.

Yameogo G, Ouedraogo H, Yelemou B. 2019. Dynamique de la biodiversité des parcs agroforestiers de Vipalogo en zone nord soudanienne du Burkina Faso. International Journal of Biological and Chemical Sciences, 13(6) : 2765-2776. DOI:

https://dx.doi.org/10.4314/ijbcs.v13i6.27 